\title{
Effectiveness of step-down versus outpatient dialectical behaviour therapy for patients with severe levels of borderline personality disorder: a pragmatic randomized controlled trial
}

\author{
Roland Sinnaeve $e^{1,3^{*+}}$, Louisa M. C. van den Bosch ${ }^{1,4+}$, Leona Hakkaart-van Roijen ${ }^{2}$ and Kristof Vansteelandt ${ }^{3}$
}

\begin{abstract}
Background: Step-down dialectical behaviour therapy (DBT) is a treatment consisting of 3 months of residential DBT plus 6 months of outpatient DBT. The program was specifically developed for people suffering from severe borderline personality disorder (BPD). The present study examines the effectiveness and cost-effectiveness of stepdown DBT compared to 12 months of regular, outpatient DBT.

Methods: Eighty-four participants reporting high levels of BPD-symptoms (mean age 26 years, 95\% female) were randomly assigned to step-down versus standard DBT. Measurements were conducted at baseline and after 3, 6, 9 and 12 months. The Lifetime Parasuicide Count and BPD Severity Index (BPDSI) were used to assess suicidal behaviour, non-suicidal self-injury (NSSI) and borderline severity. Costs per Quality Adjusted Life Year (QALY) were calculated using data from the EQ-5D-3L and the Treatment Inventory Cost in Psychiatric Patients (TIC-P).

Results: In step-down DBT, $95 \%$ of patients started the program, compared to $45 \%$ of patients in outpatient DBT. The probability of suicidal behaviour did not change significantly over 12 months. The probability of NSSI decreased significantly in step-down DBT, but not in outpatient DBT. BPDSI decreased significantly in both groups, with the improvement leveling off at the end of treatment. While step-down DBT was more effective in increasing quality of life, it also cost significantly more. The extra costs per gained QALY exceeded the $€ 80,000$ threshold that is considered acceptable for severely ill patients in the Netherlands.

Conclusions: A pragmatic randomized controlled trial in the Netherlands showed that 9 months of step-down DBT is an effective treatment for people suffering from severe levels of BPD. However, step-down DBT is not more effective than 12 months of outpatient DBT, nor is it more cost-effective. These findings should be considered tentative because of high noncompliance with the treatment assignment in outpatient DBT. Furthermore, the longterm effectiveness of step-down DBT, and moderators of treatment response, remain to be evaluated.
\end{abstract}

Trial registration: www.clinicaltrials.govNCT01904227. Registered 22 July 2013 (retrospectively registered).

Keywords: Borderline personality disorder, Dialectical behaviour therapy, Residential treatment, Suicide, Self-injurious behaviour, Randomized controlled trial, Cost effectiveness

\footnotetext{
* Correspondence: roland.sinnaeve@kuleuven.be

${ }^{\dagger}$ Roland Sinnaeve and Louisa M. C. van den Bosch contributed equally to this work.

${ }^{1}$ GGZ Rivierduinen - Centre for Personality Disorders Jelgersma,

Rhijngeesterstraatweg 13-C, 2342 AN Oegstgeest, The Netherlands

${ }^{3}$ Z.org KU Leuven, University of Leuven, Leuvensesteenweg 517, 3070

Kortenberg, Belgium

Full list of author information is available at the end of the article
}

(c) The Author(s). 2018 Open Access This article is distributed under the terms of the Creative Commons Attribution 4.0 International License (http://creativecommons.org/licenses/by/4.0/), which permits unrestricted use, distribution, and reproduction in any medium, provided you give appropriate credit to the original author(s) and the source, provide a link to the Creative Commons license, and indicate if changes were made. The Creative Commons Public Domain Dedication waiver (http://creativecommons.org/publicdomain/zero/1.0/) applies to the data made available in this article, unless otherwise stated. 


\section{Background}

\section{Outpatient dialectical behaviour therapy: an efficacious} treatment for BPD

Borderline Personality Disorder (BPD) is a severe and persistent mental disorder. Clinical hallmarks include emotional dysregulation, impulsivity, self-injury and chronic suicidal ideation [1]. The suicide rate is higher than that found in the general population [2]. A recent prospective study of the course and outcome of 290 inpatients diagnosed with BPD found a completed suicide rate of about $4 \%$ in the first 6 years of follow-up [3]. The diagnoses is associated with high burden of illness [4], poor social outcome $[5,6]$ and health provider stigma [7].

Dialectical Behaviour Therapy (DBT) was developed for chronically suicidal individuals diagnosed with BPD. The treatment strategies are rooted in Linehan's emotion regulation (skills deficit) model, which states that dysfunctional behaviour in BPD can be explained as either consequences of pervasive emotion dysregulation or ways of coping with it $[8,9]$. The first phase of DBT focusses on skills to stop the vicious circle of emotion dysregulation. Standard phase one DBT has five components. First, a weekly skills training to increase capabilities to be mindful, regulate emotions, tolerate frustration and be effective in interpersonal relationships. Groups complete the curriculum twice over the course of a year, creating a 1-year training program. Second, individual therapy to enhance motivation and to help apply DBT skills in daily life. A third component, telephone coaching, facilitates skills generalization between sessions. The fourth and fifth components include specific case management strategies and team meetings to help therapists stay motivated and competent $[8,9]$.

The efficacy of standard, outpatient DBT as a treatment for BPD has been demonstrated in randomized controlled trials (RCT) in academic settings [10-17]. Follow-up data indicated treatment gains were maintained 6 to 12 months after treatment [18-20]. It was also shown DBT remains effective when it is implemented in non-academic settings [21-24]. A meta-analysis about the effects of psychological treatments for BPD confirmed that DBT is helpful in reducing inappropriate anger and non-suicidal self-injury (NSSI) as well as in improving general functioning [25]. Research evaluating mechanisms of change found applying DBT skills in daily life mediates treatment effectiveness [26, 27].

\section{A recurring debate: inpatient and residential treatments for BPD}

DBT was originally developed in an outpatient setting $[8,11]$. Linehan argued that if the function of suicidal behaviour is the communication of distress, the desire for companionship or avoidance of some aversive reality in daily life, then being hospitalized may reinforce the suicidal behaviour and prevent patients from developing functional coping skills to address their problems [8]. At the same time, longitudinal studies demonstrate that patients diagnosed with BPD are more likely to be hospitalized than patients diagnosed with other mental disorders [28-30]. Bloom et al. argued some patients are not sufficiently engaged in outpatient treatment and exacerbations of symptoms can exceed what providers can manage in an outpatient setting [31], suggesting the importance of effective inpatient DBT. Bloom et al. synthesized findings from 11 pre-post studies on the efficacy of inpatient DBT. Most studies reported reductions in suicidal ideation, self-injurious behaviour and symptoms of depression and anxiety. However, caution is needed when interpreting these findings as none of the studies were RCT's, few included a comparison group and most were plagued by sample size issues [31].

\section{Synthesis: residential treatment as a preparation for outpatient treatment?}

Bloom et al. stated that examining the effectiveness of inpatient DBT as an intensive preparation for outpatient DBT is the next step in developing best-practice guidelines [31]. Along the same lines, we wondered if it was possible to improve the effectiveness of DBT by developing a step-down DBT-program using 3 months of residential DBT as an intensive orientation to 6 months of outpatient DBT [32]. We use the term 'residential' instead of 'inpatient' to clarify that the residence was a home-like environment where patients only stayed on weekdays [33]. Support staff were only present during office hours. The residential setting allowed us to adapt standard DBT protocol: DBT skills were trained in 3 months instead of 6 months, patients were reminded about their skills every weekday and extra program parts, aimed at practicing and generalizing skills, were added. We hypothesized that for individuals suffering from high levels of BPD-symptoms this 9-month, step-down DBT program would lead to a significantly larger decrease in suicidal behaviour, NSSI and total level of borderline symptomatology than 12 months of standard DBT. We also expected that step-down DBT would lead to fewer drop-outs and would be more cost-effective when estimated over a 12-month period [32]. To our knowledge, this is the first time that these hypotheses were evaluated in a randomized controlled trial.

\section{Methods}

We conducted an RCT with a two (group) by five (time) repeated measures parallel design, without blinding. The sequence of randomization was concealed until interventions were assigned. The protocol was in accordance to the principles outlined in the Declaration of Helsinki, approved by the Institutional Review Board and registered in www.clinicaltrials.gov [32]. There are three differences between the study protocol in Trials and this report. First, 
the name of the residential program was changed from 'inpatient DBT' to 'residential DBT'. Second, our study ended prematurely due to an unexpected close-down of the Centre for Personality Disorders Jelgersma (CPJ). Third, because of unforeseen waiting list issues, participants who were randomized to outpatient DBT had to wait longer before they met their therapist.

\section{Sample}

Participants gave written informed consent. They had to meet the DSM-IV TR criteria for BPD (identical to the criteria in DSM-5), be 18-45 years of age, score higher than 24 on the Borderline Severity Index-IV (BPDSI-IV) and report at least one episode of self-injurious behaviour within the month before the intake. If there was no episode of self-injurious behaviour 1 month before the intake, then a BDSI-score of at least 30 was required to be eligible for the study. Exclusion criteria were limited to having a diagnosis of a chronic psychotic disorder, bipolar I disorder, intellectual disability, substance dependence requiring detoxification, involuntary psychiatric treatment, insufficient command of Dutch or living outside of travelling distance from the treatment center.

\section{Therapists and trainers}

All therapists and trainers were psychologists, psychiatrists, nurses or social workers working at GGZ Rivierduinen $(n=30)$. DBT team members completed at minimum a 3-day training in DBT and received supervision from the senior researcher. Adherence was assessed with the 5-point DBT Expert Rating Scale (Linehan, Lockard, Wagner \& Tutek: DBT Expert Rating Scale, unpublished). Treatment integrity greater than or equal to four was considered adherent. Fifteen percent of the sessions were assessed. Scores ranged between 3.6 and 4.1, with an average of 3.9. Both step-down DBT and outpatient DBT contained the five components of the treatment protocol $[8,11,12]$. The DBT-skills were taught according to the first version of the manual $[8$, 12]. The only adaption was that telephone consultation outside of the office hours was within the limitations set by the therapist.

\section{Treatments}

The experimental treatment, step-down DBT, consisted of 3 months of residential DBT plus 6 months of outpatient DBT [32]. In residential DBT, support staff were present during office hours to help the patients apply DBT skills. Program parts were added, including: daily mindfulness classes, daily meetings about living together as a group, weekly drama therapy, weekly group sessions on validation skills and chain analyses, and fortnightly network training sessions together with family and friends. Limiting residential DBT to 3 months had several advantages, including enabling us to limit costs, make clear to participants that the goal was preparing for outpatient DBT and to compare our results with 'modal inpatient DBT' [31, 34, 35]. Controls received 12 months of standard, outpatient DBT, organized in three community mental health settings of GGZ Rivierduinen [32].

\section{Measurements \\ Intake interview}

Participants were screened with the Vragenlijst Kenmerken Persoonlijkheid [36]. Presence of Axis 1 and Axis 2 disorders was assessed with the mini-International Neuropsychiatric Interview [37] and the Structured Clinical Interview for DSM Disorders [38]. These are DSM IV-TR diagnoses. Validated, semi-structured interviews for DSM 5 diagnoses were not available in Dutch. A Dutch translation of the Lifetime Parasuicide Count (LPC) was used to obtain detailed information about the nature, frequency and function of self-injurious behaviour (Comtois \& Linehan: Lifetime Parasuicide Count: description and psychometrics, unpublished; van den Bosch: Vragenlijst Parasuicidaal gedrag, unpublished). The LPC makes a distinction between self-injurious behaviour with suicidal intentions (LPC Sui), without suicidal intentions (LPC NSSI) or ambivalent suicidal intentions (LPC Amb). Frequency of borderline symptoms in the previous 3-month period was assessed with the BPDSI-IV [39].

\section{Repeated assessments}

After randomization, assessments took place at baseline and after 3, 6, 9 and 12 months. The LPC and BPDSI were used to collect data on suicidal behaviour, NSSI and borderline severity in the past 3 months. Quality of life was assessed with the EQ-5D 3 level version (EQ-5D-3L). The health descriptions of this measure can be linked directly to empirical valuations of the general public, which allows utilities to be computed [40]. The Dutch tariff was used to calculate preferences for EQ-5D health states [41]. Direct medical costs and productivity costs were measured with the Treatment Inventory Cost in Psychiatric Patients [TiC-P] [42]. Unit costs were valued according to prices reported in the Dutch manual for cost research [43].

\section{Randomization}

A computer program, developed by the Amsterdam Medical Centre, generated the sequence. To increase the likelihood of comparable treatment groups, a minimization method was used. Minimization variables were BPDSI score $\geq 40$, total lifetime LPC score $\geq 14$ and age.

\section{Statistical analyses}

Comparisons of key demographic and clinical characteristics of the analyzed sample were performed with t-tests 
for normally distributed variables and with Wilcoxon two sample tests for variables that were not normally distributed. To examine the association between categorical variables, we used Chi-square tests and Fisher's exact tests. In the repeated measurements analyses data from the LPC, subscales were dichotomized because they were right-skewed with excess zeros. A generalized linear mixed model (GLMM) with random intercepts with logistic link function was estimated for dichotomous outcome variables [44]. This allowed us to examine whether the probability (yes or no) of self-destructive behaviour changed over time. A linear mixed model (LMM) with random intercepts and slopes was estimated for the BPDSI total score. In these models, condition, time, and time $\mathrm{x}$ condition were included as fixed effects. Time was expressed as number of months passed since baseline. Model selection and inference were based on Likelihood Ratio and Wald tests [45]. The Kaplan-Meier statistic was used to examine whether the time to dropout was longer for patients in step-down DBT compared to outpatient DBT. Non-starters were excluded from this analysis.

The cost-effectiveness of step-down DBT was assessed by estimating an incremental cost-effectiveness ratio (ICER). In this case, the ICER was the difference in costs of both interventions divided by the difference in quality adjusted life years gained (QALY's). QALY's were estimated using the EQ-5D-3L scores. Details of procedures to calculate the direct medical costs, productivity costs (e.g. absenteeism and presenteeism) and the ICER can be found in the guidelines of costing studies [43]. Subsequently, we assessed the probability that step-down DBT is more effective than outpatient DBT by comparing the costs per QALY to the costs that are considered acceptable for severely ill patients in the Netherlands (i.e. $€$ $80,000)$ [46]. Non-parametric tests were conducted since the data were not normally distributed. A bootstrap simulation was run for 5000 iterations to estimate $95 \%$ confidence intervals (CI) for a range of probable values for total costs, effects and ICERs.

\section{Results}

\section{Participant flow}

A total of 187 participants were assessed for eligibility from February 2012 to January 2014 (Fig. 1). Sixty-three participants did not meet the inclusion criteria. After randomization, two out of 42 participants in step-down DBT did not start the allocated treatment and one participant did not provide valid baseline data. Consequently, 39 participants were included in the primary analyses. In outpatient DBT, 23 out of 42 participants did not start the allocated treatment. This could be partially due to the fact that waiting times appeared to be longer in outpatient DBT. One participant died by suicide before he received outpatient DBT. Three out of 19

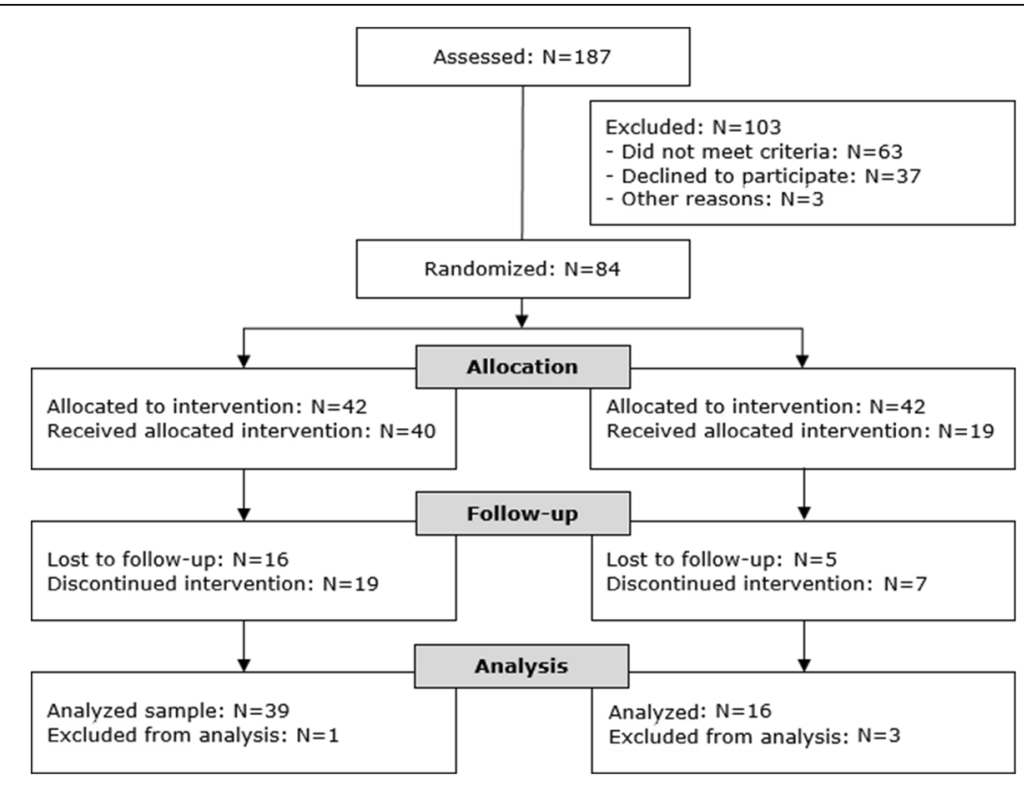

Fig. 1 CONSORT flowchart pragmatic RCT step-down versus outpatient DBT. 'Received the allocated intervention' = Number of participants that attended at least one skills training or at least one individual therapy session after they signed a therapist-client agreement (referred to as 'starters' in the text). 'Lost to follow up'= Number of participants that received the allocated intervention but did not complete a 12 month assessment. 'Discontinued intervention' = Number of participants that received the allocated intervention but dropped out before it was completely finished. In DBT, 'dropout' means that a participant missed four individual therapy or four weekly skills training session in a row. 'Analyzed' = Number of participants whose data were used to estimate statistical models for primary outcome variables 
participants that started outpatient DBT did not provide valid baseline data.

\section{Characteristics analysed sample}

The sample was $95 \%$ female. Fourteen participants were working (26\%). An equal number was enrolled in college. Nineteen participants $(35 \%)$ were considered unfit for work. The majority $(N=47,85.5 \%)$ was divorced or single. Almost one third of the sample reported a history of sexual abuse $(N=16,29 \%)$ and more than half experienced physical abuse $(N=30,55 \%)$. One out of three participants suffered from posttraumatic stress disorder $(N=17,31 \%)$, half were diagnosed with major depression $(N=28,51 \%)$, and one out of three participants fulfilled criteria of substance dependence $(N=17,31 \%)$. The average EQ-5D-3L score at baseline was .47 [standard deviation $(\mathrm{SD})=.29$ ], confirming an overall low quality of life. The total direct medical costs in the year before the study were high. The main cost drivers were admissions to psychiatric hospitals $[€ 16,248$ (SD = $€ 32,838)]$ and psychotherapy [€5274 (SD = €7662)]. Other characteristics can be found in Table 1. There were no significant between-group differences in key demographic or clinical variables.

\section{Outcomes}

\section{Suicidal behaviour and NSSI}

No completed suicides were recorded after participants started DBT. The probability of self-injurious behaviour with suicidal intention (LPC Sui), $\mathrm{F}(2,156)=2.90, p$ $=.06$, and with ambivalent suicidal intention (LPC $\mathrm{Amb}), \mathrm{F}(2,156)=2.63, p=.08$, did not change significantly over 12 months. We found that the probability of self-injurious behaviour with suicidal intention, odds ratio $(\mathrm{OR})=.33,95 \% \mathrm{CI}[.17-.63], \mathrm{F}(1,32)=12.28, p=.001$, and with ambivalent suicidal intention, $\mathrm{OR}=.55,95 \% \mathrm{CI}$ [.38-.81], $\mathrm{F}(1,32)=10.00, p=.003$, decreased during the 3 months of residential treatment in the step-down DBT group. This means that the hypothesis that a residential setting reinforces suicidal behaviour was rejected.

There were significant changes in the probability of NSSI (LPC NSSI) during treatment, $\mathrm{F}(2,156)=4.27$, $p=.02$. More specifically, the probability of NSSI decreased significantly over 12 months in step-down $\mathrm{DBT}, \mathrm{OR}=.90,95 \% \mathrm{CI}[.82-.98], \mathrm{t}(156)=-2.45, p=.02$, but not in outpatient DBT, OR $=.90,95 \%$ CI [.79-1.03], $\mathrm{t}(156)=-1.60, p=.11$. Note that the difference between both groups is small. The fact that the OR is not significant in the outpatient DBT may be due to its smaller sample size. The estimated probabilities of self-injurious episodes during step-down DBT and outpatient DBT can be found in Table 2. The mean frequency of self-injurious episodes can be found in Table 3.

\section{Drop-out}

In step-down DBT, $53 \%$ of the participants who started DBT finished the entire 9-month program. Twelve months of outpatient DBT showed a retention rate of $63 \%$. The results of the Kaplan Meier statistic indicated that there were no significant differences in the time to drop-out between conditions, $\mathrm{X}^{2}(1)=.36, p=.55$.

\section{Severity of $B P D$}

The BPDSI total-score indicated that borderline symptomatology decreased significantly in both treatment groups, $\mathrm{F}(1,109)=33.63, p<.0001$. The regression coefficients for months in step-down DBT and outpatient DBT were respectively $-2.87(\mathrm{SE}=.37), \mathrm{t}(109)$ $=-7.86, p<.001$, and $-2.82(\mathrm{SE}=.41), \mathrm{t}(109)=-6.82$, $p<.0001$ (Table 4). This decrease levelled off near the end of treatment, $\mathrm{F}(1,109)=23.92, p<.0001$. The regression coefficient of the quadratic effect of months was .1 (SE =.03) (Fig. 2).

Table 1 Comparisons of key demographic and clinical characteristics in step-down DBT and outpatient DBT

\begin{tabular}{|c|c|c|c|c|c|c|c|}
\hline & \multicolumn{2}{|c|}{ Step-down DBT ${ }^{\mathrm{a}}$} & \multicolumn{2}{|c|}{ Outpatient DBT ${ }^{\mathrm{b}}$} & \multirow{2}{*}{$\begin{array}{l}\text { Test } \\
\text { Statistic }\end{array}$} & \multirow[t]{2}{*}{$d f$} & \multirow[t]{2}{*}{$p$} \\
\hline & $\mathrm{M}$ & SE & $\mathrm{M}$ & SE & & & \\
\hline Age first mental health problems & 11.13 & .79 & 11.88 & .67 & $t=-.72$ & 49 & .47 \\
\hline Age first contact mental health care & 14.28 & 1.15 & 15.44 & 1.20 & $t=-.59$ & 53 & .56 \\
\hline Number of BPD criteria & 7.10 & .20 & 7.56 & .30 & $t=-1.25$ & 53 & .22 \\
\hline Severity of BPD $3 \mathrm{~m}$ before intake & 37.17 & 1.27 & 34.75 & 1.29 & $t=1.12$ & 53 & .27 \\
\hline Age at time of intake & 26.15 & $6.18^{d}$ & 25.63 & $7.45^{\mathrm{d}}$ & $Z=-.71^{e}$ & n.a. & .48 \\
\hline Suicide attempts lifetime & 4.72 & $6.79^{d}$ & 25.84 & $52.90^{\mathrm{d}}$ & $Z=-.64^{e}$ & n.a. & .52 \\
\hline Ambivalent SI lifetime $(N=54)$ & $12.50^{c}$ & $28.76^{d}$ & 33.88 & $77.59^{d}$ & $\mathrm{Z}=.41^{\mathrm{e}}$ & n.a. & 68 \\
\hline NSSI lifetime $(N=44)$ & $525.48^{c}$ & $876.74^{d}$ & $938.07^{c}$ & $1644.26^{d}$ & $Z=-.23^{e}$ & n.a. & .82 \\
\hline
\end{tabular}

n.a. not applicable; Comparisons performed with t-tests for normally distributed variables and with Wilcoxon two sample tests for variables that were not normally distributed. ${ }^{\mathrm{a}} \mathrm{N}=39 ;{ }^{\mathrm{b}} \mathrm{N}=16 ;{ }^{\mathrm{c}}$ Patients that persisted that they could not recall lifetime occurrence of self-injurious behaviours, because it was "too high to estimate"

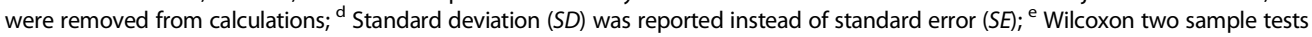


Table 2 Probabilities and 95\% confidence intervals of self-injurious episodes based on a generalized linear mixed model

\begin{tabular}{|c|c|c|c|c|c|c|}
\hline & \multicolumn{3}{|c|}{ Step-down DBT ${ }^{a}$} & \multicolumn{3}{|c|}{ Outpatient DBT } \\
\hline & LPC Sui & LPC Amb & LPC NSSI & LPC Sui & LPC Amb & LPC NSSI \\
\hline \multirow[t]{2}{*}{ Baseline } & .18 & .26 & .80 & .29 & .09 & .80 \\
\hline & [.09-.33] & {$[.15-.42]$} & {$[.65-.89]$} & {$[.11-.58]$} & {$[.02-.29]$} & {$[.56-.93]$} \\
\hline \multirow[t]{2}{*}{$0-3 \mathrm{~m}$} & .17 & .20 & .74 & .17 & .11 & .75 \\
\hline & {$[.09-.27]$} & {$[.12-.31]$} & {$[.61-.84]$} & {$[.07-.36]$} & [.04-.28] & {$[.54-.88]$} \\
\hline \multirow[t]{2}{*}{$3-6 m$} & .15 & .15 & .67 & .09 & .14 & .69 \\
\hline & {$[.09-.25]$} & {$[.09-.25]$} & {$[.54-.78]$} & {$[.03-.24]$} & [.06-.29] & {$[.49-.83]$} \\
\hline \multirow[t]{2}{*}{$6-9 m$} & .13 & .11 & .60 & .05 & .17 & .62 \\
\hline & {$[.07-.25]$} & {$[.05-.22]$} & {$[.44-.74]$} & {$[.01-.19]$} & {$[.07-.35]$} & {$[.40-.80]$} \\
\hline \multirow[t]{2}{*}{ 9-12 m } & .12 & .08 & .52 & .02 & .21 & .54 \\
\hline & {$[.05-.27]$} & {$[.03-.21]$} & {$[.33-.71]$} & {$[.003-.16]$} & {$[.07-.47]$} & {$[.28-.78]$} \\
\hline
\end{tabular}

Timeframe = 3 months before measurement; Note: LPC Sui, LPC Amb, LPC NSSI = self-injury with respectively suicidal, ambivalent, non-suicidal intentions according to Lifetime Parasuicide Count; ${ }^{\text {a }}$ Observations: $N($ Baseline $)=39, N(0-3 \mathrm{~m})=33, N(3-6 \mathrm{~m})=25, N(6-9 \mathrm{~m})=22, N(9-12 \mathrm{~m})=24$; $^{\text {b }}$ Observations: $N($ Baseline $)=16, N(0-3 \mathrm{~m})=$ $15, N(3-6 \mathrm{~m})=15, N(6-9 \mathrm{~m})=14, N(9-12 \mathrm{~m})=14$

\section{Costs and cost-effectiveness}

After 12 months, the average EQ-5D-3L score was .65 $(\mathrm{SD}=.33)$ in step-down DBT and $.62(\mathrm{SD}=.28)$ in outpatient DBT. In step-down DBT, healthcare costs were higher: $€ 19,899 \quad(\mathrm{SD}=14,210)$ versus $€ 12,472 \quad(\mathrm{SD}=$ $14,300)$. There were no differences in productivity costs, with $€ 906$ (SD = 3462) for step-down DBT and €964 (SD = 3633) for outpatient DBT. The ICER with imputed values was $€ 278,067$ per QALY. The acceptability curve showed that the intervention has a probability of $21 \%$ of being cost-effective if the maximum threshold is $€$ 80,000 . The ICER was recomputed to gauge the effect of the imputation process on the underlying data. The ICER was reduced to $€ 220,566$, which is still above the threshold. The bootstrap-data are shown in Fig. 3. The majority of the points (59\%) lie in the north-east quadrant of the CE-plane. This indicates that step-down DBT is more effective in increasing quality of life, but also more costly, than outpatient DBT.

Table 3 Mean frequency and standard deviation of self-injurious episodes

\begin{tabular}{|c|c|c|c|c|c|c|c|c|c|c|c|c|}
\hline & \multicolumn{6}{|c|}{ Step-down DBT ${ }^{a}$} & \multicolumn{6}{|c|}{ Outpatient DBT } \\
\hline & \multicolumn{2}{|c|}{ LPC Sui } & \multicolumn{2}{|c|}{ LPC Amb } & \multicolumn{2}{|c|}{ LPC NSSI } & \multicolumn{2}{|c|}{ LPC Sui } & \multicolumn{2}{|c|}{ LPC Amb } & \multicolumn{2}{|c|}{ LPC NSSI } \\
\hline & $\bar{M}$ & $\overline{S D}$ & M & SD & $\bar{M}$ & SD & $\mathrm{M}$ & $S \mathrm{SD}$ & M & SD & $\mathrm{M}$ & SD \\
\hline & 1.0 & 2.4 & 1.9 & 4.5 & 36.1 & 54.5 & 1.2 & 2.7 & 1.9 & 4.0 & 41.7 & 23.2 \\
\hline (11 & .0 & .2 & 3.7 & 13.5 & 13.6 & 34.5 & .1 & .3 & 1.0 & 3.9 & 23.2 & 53. \\
\hline & 1.2 & 4.2 & 1.4 & 6.0 & 23.3 & 52.6 & .1 & .3 & .5 & 1.1 & 15.7 & 30.8 \\
\hline$-9 m$ & .8 & 3.2 & 1.0 & 2.7 & 17.0 & 29.2 & .2 & 6 & 1.3 & 4.5 & 7.3 & 14.3 \\
\hline $12 \pi 11$ & .2 & .7 & .0 & .2 & 4.5 & 9.0 & .5 & 1.9 & .9 & 2.1 & 8.6 & 14.0 \\
\hline
\end{tabular}

LPC Sui, LPC Amb, LPC NSSI = self-injury with respectively suicidal, ambivalent, non-suicidal intentions according to Lifetime Parasuicide Count; $M$ mean, $S D$ standard deviation; ${ }^{a}$ Observations: $N($ Baseline $)=39, N(0-3 \mathrm{~m})=33, N(3-6 \mathrm{~m})=25$, $N(6-9 \mathrm{~m})=22, N(9-12 \mathrm{~m})=24$; ${ }^{\mathrm{b}}$ Observations: $N($ Baseline $)=16, N(0-3 \mathrm{~m})=15, N(3-$ $6 \mathrm{~m})=15, N(6-9 \mathrm{~m})=14, N(9-12 \mathrm{~m})=14$

\section{Discussion}

We conducted a pragmatic RCT to compare 9 months of step-down DBT to 12 months of outpatient DBT in a sample that reported severe levels of BPD. Step-down DBT consisted of 3 months of residential plus 6 months of outpatient DBT. Our main findings were that: a) the probability of suicidal behaviour did not change significantly over 12 months, b) the probability of NSSI decreased significantly in step-down DBT, but not in outpatient DBT, c) severity of borderline symptomatology decreased significantly in both groups, with the improvement leveling off at the end of treatment, and d) the extra costs per gained QALY in step-down DBT exceeded the $€ 80,000$ threshold that is considered acceptable for severely ill patients in the Netherlands.

In the step-down program, 40 out of 42 (95\%) patients were willing to initiate DBT treatment. In the outpatient program, only 19 out of 42 (45\%) patients were willing to initiate DBT treatment. The noncompliance in outpatient DBT may have introduced confounding. We reported that participants who were randomized to outpatient DBT had to wait longer before they met their therapist. It is possible that participants who were willing

Table 4 Borderline Personality Disorder Symptom Index: estimated means based on a linear mixed model

\begin{tabular}{|c|c|c|c|c|c|c|}
\hline & \multicolumn{3}{|c|}{ Step-down DBT ${ }^{a}$} & \multicolumn{3}{|c|}{ Outpatient DBT } \\
\hline & $\bar{M}$ & SE & $95 \% \mathrm{Cl}$ & $M$ & SE & $95 \% \mathrm{Cl}$ \\
\hline Baseline & 35.80 & 1.38 & $33.07-38.52$ & 32.27 & 2.14 & $28.02-36.52$ \\
\hline $0-3 \mathrm{~m}$ & 28.32 & 1.38 & $25.58-31.06$ & 24.97 & 2.10 & 20.81-29.13 \\
\hline $3-6$ m & 23.14 & 1.68 & $19.82-26.47$ & 19.97 & 2.41 & $15.19-24.75$ \\
\hline 6-9 m & 20.27 & 1.98 & $16.33-24.20$ & 17.27 & 2.87 & $11.58-22.95$ \\
\hline 9-12 m & 19.69 & 2.44 & $14.85-24.52$ & 16.86 & 3.51 & $9.91-23.82$ \\
\hline
\end{tabular}

$M$ mean, $S E$ standard error, $\mathrm{Cl}$ confidence interval; ${ }^{a}$ Observations: $N($ Baseline $)=$ $39, N(0-3 \mathrm{~m})=33, N(3-6 \mathrm{~m})=25, N(6-9 \mathrm{~m})=22, N(9-12 \mathrm{~m})=24{ }^{\mathrm{b}}$ Observations: $N($ Baseline $)=16, N(0-3 \mathrm{~m})=15, N(3-6 \mathrm{~m})=15, N(6-9 \mathrm{~m})=14, N(9-12 \mathrm{~m})=14$ 


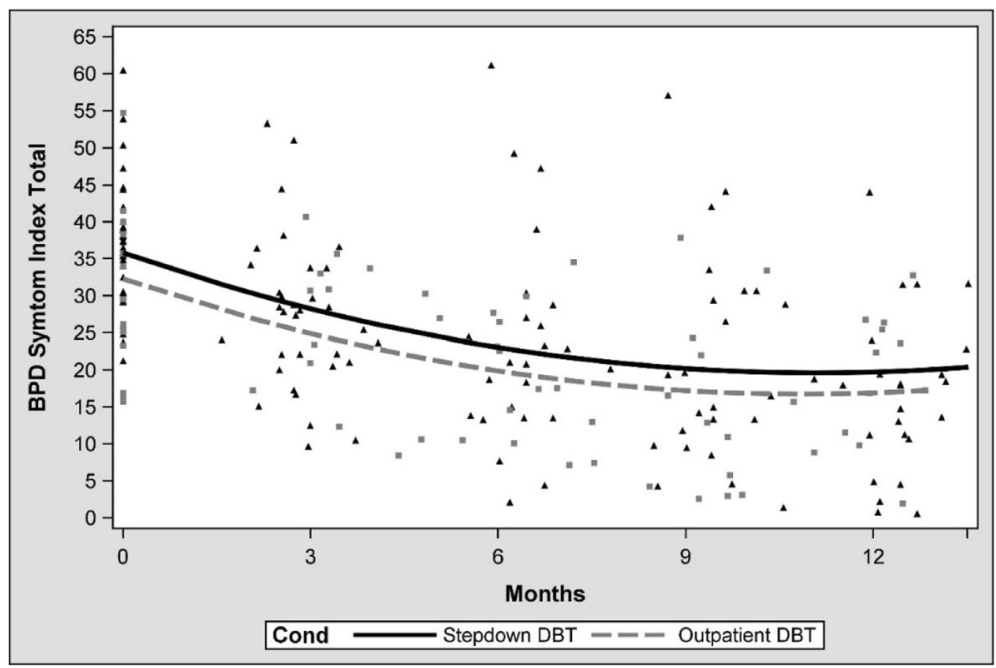

Fig. 2 Estimated Borderline Personality Symptom Index score with time, condition and time $x$ condition as predictors

to wait, differed systematically from those who refrained from treatment or sought help elsewhere. Alternatively, it is plausible that step-down DBT reached a subsample that was unable to engage in an outpatient treatment [31]. However, we found no significant differences on key demographic or clinical variables between the starters in step-down DBT and outpatient DBT. On balance, the fact that initial randomization was undermined, poses a threat to the internal validity of our study. This means that the main findings should be considered tentative. In other words, the results of our study do not warrant shifting resources from step-down to outpatient DBT programs.

We also tested the hypothesis that a residential setting reinforces suicidal behaviour. This hypothesis was rejected. On the contrary, we found a significant decrease in the probability of suicidal behaviour during the first 3 months of step-down DBT (i.e. the residential phase). These findings are similar to those reported in studies of 3-month inpatient DBT [34, 47], and challenge the perspective that hospitalization always reinforces suicidal behaviour in people diagnosed with BPD. It seems that possible iatrogenic effects of hospitalization can be neutralized if the support staff is trained in DBT. Of note, only $35 \%$ of participants still engaged in NSSI after Bohus' inpatient DBT-program [34]. In our residential DBT-program over $70 \%$ of the participants reported that they still engaged in NSSI during treatment. Percentages at baseline were almost identical: about $75 \%$ $[34,47]$. This difference may be related to the timeframe that was used to measure NSSI. In Bohus et al., participants were asked to report NSSI that occurred in the last month. In our study, participants were asked to report NSSI that occurred in the last 3 months. Another plausible explanation is the difference in coaching after office hours. The hospital setting in Bohus et al. allowed for 24/7 crisis interventions by support staff. In our study, support staff were only present during office

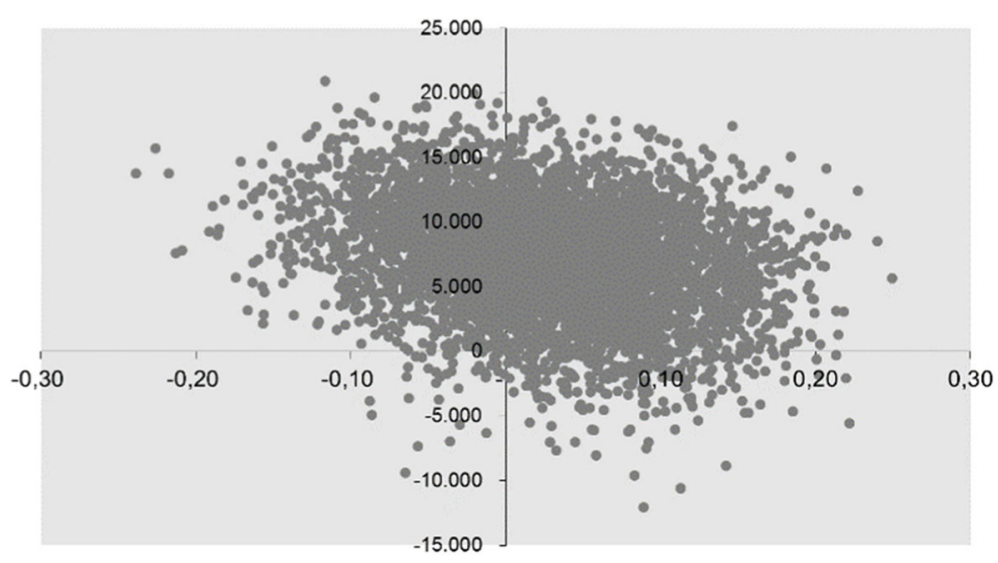

Fig. 3 Cost-effectiveness plane step-down DBT versus outpatient DBT. The Y-axis represents additional effects. The X-axis represents additional costs 
hours and on weekdays. Telephone consultation after office hours was within the limitations set by the therapist. Future research should take this into account, either by implementing this component of DBT as well, or by conducting a dismantling study first.

Some strengths of the present study are notable. First, we ensured that interventions were allocated by means of a concealed randomization procedure. Second, treatment adherence was evaluated in both conditions. Third, the protocol was published in advance and all analyses were performed by independent experts [32]. Fourth, our design has strong ecological validity given that it was performed in a nonacademic context. On the other hand, this project had several limitations. Foremost, the initial randomization was undermined by a high percentage of non-starters in outpatient DBT. Second, data collectors were not blind to the assigned intervention. Third, the skills training groups of outpatient DBT contained patients that did not participate in the study. Thus, the composition of the skills training groups in outpatient DBT differed from the groups in step-down DBT, which consisted only of study participants. Fourth, evaluation of treatment integrity showed that some sessions were non-adherent (DBT Expert Rating Scale scores < 4.0). A final limitation is the lack of follow-up data.

The effectiveness of step-down versus outpatient DBT for patients that report severe levels of BPD-symptoms remains to be established in future research. It will be equally important to assess which moderators (e.g. characteristics of the individual or his/her social context, treatment integrity, regional differences in mental health care organization and stigma) change the direction or strength of the relation between the treatment (step-down versus outpatient) and the outcome (e.g. NSSI, BPDSI, drop-out, QALY). Given the treatment outcomes we reported in this pragmatic RCT, treatment integrity, in particular, deserves further examination. Adherence to a protocol is essential for internal validity and generalizability of the results in our domain of research. However, it would be interesting to learn more about the relationship between treatment adherence and treatment outcome. Is it a linear relationship? Or does the added value lessen once a certain level of adherence is reached? Finally, yet importantly: the long-term effectiveness and cost-effectiveness of step-down DBT remain to be evaluated.

The main methodological challenges we encountered were noncompliance and attrition in outpatient DBT. We do not know whether these phenomena indicate that step-down DBT was more effective at engaging people suffering from severe levels of BPD [31]. To answer this question in future research, we need to rule out waiting list issues and strengthen participants' commitment before randomization takes place. When these conditions are met, higher compliance in step-down DBT would provide support for Bloom's hypothesis [31]. A second step would be to find out what predicts compliance in step-down and in outpatient DBT. We found no significant differences in demographic or clinical variables in our study. Perhaps factors that we did not include, such as social isolation, institutionalization, and marginalization, were paramount. A last consideration is that, even though interesting in its own right, noncompliance challenges the feasibility and validity of a RCT. We would suggest future researchers to consider a Zelen's design, or to add a second control condition that would allow us to compare step-down DBT to 'residential care as usual' plus outpatient DBT [48].

\section{Conclusions}

A pragmatic randomized controlled trial in the Netherlands showed that 9 months of step-down DBT is an effective treatment for people suffering from severe levels of BPD. However, step-down DBT was not more effective than 12 months of outpatient DBT, nor was it more cost-effective. These findings should be considered tentative because of relatively high noncompliance with the treatment assignment in outpatient DBT. Furthermore, the long-term effectiveness of step-down DBT, and moderators of treatment response, remain to be evaluated.

\section{Abbreviations \\ BPD: Borderline Personality Disorder; BPDSI: Borderline Personality Disorder Severity Index; DBT: Dialectical Behaviour Therapy; DSM: Diagnostic Statistical Manual of Mental Disorders; GLMM: Generalized Linear Mixed Model; ICER: Incremental Cost-Effectiveness Ratio; LMM: Linear Mixed Model; LPC: Lifetime Parasuicide Count; NSSI: Non-Suicidal Self-Injury; QALY: Quality Adjusted Life Year; RCT: Randomized Controlled Trial; SCID: Structured Clinical Interview for DSM Disorders; TIC-P: Treatment Inventory Cost in Psychiatric Patients}

\section{Acknowledgements}

We thank all the members of the research team for their great effort: Vera Weber, Jet de Veij Mestdagh, Marieke de Wijn - van Leeuwen, Svea Sonneveld, Elsbeth Smedes, Clazien Bouwmans and Laura Agterberg. In addition, we thank the DBT -teams for their commitment to deliver qualitative treatment for these patients. We also want to thank the clinical directors, Drs. M. Nijs and Prof. Dr. E.F. van Furth, for their support. Furthermore, we would like to thank Dr. Maarten Koeter for his help with the pilot study, and Lynnaea Northey, Dr. Andrew White, and Dr. Darren Courtney for their feedback on the final version of the manuscript. We also want to thank the anonymous reviewers for their careful reading of our manuscript and their insightful comments and suggestions. Finally, yet importantly, we thank all the participants in the trial.

\section{Funding}

GGZ Rivierduinen provided financial support to collect the data. Our project did not receive any other specific grants from funding agencies in the public, commercial, or non-profit sectors.

\section{Availability of data and materials}

The datasets used and/or analyzed during the current study are available from the corresponding author on reasonable request.

\section{Authors' contributions}

LMCB conceived of the research questions and designed the study. LMCB and RS oversaw the data acquisition. $\mathrm{LH}$ and $\mathrm{KV}$ analyzed the data. LMCB, RS, $\mathrm{KV}$, and $\mathrm{LH}$ interpreted the data. RS and LMCB drafted the report. All contributors provided critical revisions to the report, important intellectual content, and final approval. 


\section{Ethics approval and consent to participate}

This study was approved by the Medical Ethics Committee of the Leiden University Medical Center (P11.012), the Institutional Scientific Board of Rivierduinen and the boards of the participating sites. All participants gave written informed consent.

\section{Consent for publication}

Written informed consent was obtained from the participant for publication of their individual details in this manuscript. The consent form is held by the authors' institution and is available for review by the Editor-in-Chief.

\section{Competing interests}

L.M.C.B. is shareholder of Dialexis, the training institute for Dialectical Behaviour Therapy (DBT) in the Netherlands. R.S. works for Dialexis as an instructor. The authors declare that they have no competing interests.

\section{Publisher's Note}

Springer Nature remains neutral with regard to jurisdictional claims in published maps and institutional affiliations.

\section{Author details}

'GGZ Rivierduinen - Centre for Personality Disorders Jelgersma,

Rhijngeesterstraatweg 13-C, 2342 AN Oegstgeest, The Netherlands. ${ }^{2}$ Erasmus School of Health Policy and Management (ESPHM) and institute for Medical Technology Assessment (iMTA), Erasmus University Rotterdam, Postbus 1738, 3000 DR Rotterdam, The Netherlands. ${ }^{3}$ Z.org KU Leuven, University of Leuven, Leuvensesteenweg 517, 3070 Kortenberg, Belgium. ${ }^{4}$ Present address: Scelta, Deventerstraat 459, 7323 PT Apeldoorn, The Netherlands.

\section{Received: 13 March 2018 Accepted: 26 June 2018}

\section{Published online: 10 July 2018}

\section{References}

1. American Psychiatric Association. Diagnostic and statistical manual of mental disorders (DSM5). 5th ed. Arlington: American Psychiatric Publishing; 2013.

2. Gunderson JG. Borderline personality disorder: a clinical guide. Washington, DC: American Psychiatric Publishing; 2001.

3. Zanarini MC, Frankenburg FR, Hennen J, Reich DB, Silk KR. The McLean study of adult development (MSAD): overview and implications of the first six years of prospective follow-up. J Personal Disord. 2005;19(5):505-23.

4. Soeteman DI, Hakkaart-van Roijen L, Verheul R, Busschbach JJV. The economic burden of personality disorders in mental health care. J Clin Psychiatry. 2008;69(2):259-65.

5. Weinstein Y, Gleason MEJ, Oltmanns TF. Borderline but not antisocial personality disorder symptoms predict self-reported partner aggression in later life. J Abnorm Psychol. 2012;121(3):692-8.

6. Zanarini MC, Frankenburg FR, Reich DB, Fitzmaurice G. The 10-year course of psychosocial functioning among patients with borderline personality disorder and axis II comparison subjects. Acta Psychiatr Scand. 2010;122(2):103-9.

7. Sheehan L, Nieweglowski K, Corrigan P. The stigma of personality disorders. Curr Psychiatry Rep. 2016;18(1):1-7.

8. Linehan MM. Cognitive-behavioral treatment of borderline personality disorder. New York: Guilford Press; 1993

9. Linehan MM. DBT skills training manual. 2nd ed. New York: Guilford Press; 2015.

10. Clarkin JF, Levy KN, Lenzenweger MF, Kernberg OF. Evaluating three treatments for borderline personality disorder: a multiwave study. Am J Psychiatry. 2007;164(6):922-8

11. Linehan MM, Armstrong HE, Suarez A, Allmon D, Heard HL. Cognitivebehavioral treatment of chronically parasuicidal borderline patients. Arch Gen Psychiatry. 1991;48(12):1060-4.

12. Linehan MM, Comtois KA, Murray AM, Brown MZ, Gallop RJ, Heard HL, et al. Two-year randomized controlled trial and follow-up of DBT vs therapy by experts for suicidal behaviors and BPD. Arch Gen Psychiatry. 2006:63(7):757-66.

13. Linehan MM, Dimeff LA, Reynolds SK, Comtois K, Shaw-Welch S, Heagerty $P$, et al. Dialectical behavior therapy versus comprehensive validation therapy plus 12-step for the treatment of opioid dependent women meeting criteria for borderline personality disorder. Drug Alcohol Depend. 2002;67(1):13-26.
14. Linehan MM, Korslund KE, Harned MS, Gallop RJ, Lungu A, Neacsu AD, et al. Dialectical behavior therapy for high suicide risk in individuals with borderline personality disorder: a randomized clinical trial and component analysis. JAMA Psychiatry. 2015;72(5):475-82.

15. Linehan MM, Schmidt H, Dimeff LA, Craft JC, Kanter J, Comtois KA. Dialectical behavior therapy for patients with borderline personality disorder and drug-dependence. Am J Addict. 1999;8(4):279-92.

16. McMain SF, Links PS, Gnam WH, Guimond T, Cardish RJ, Korman L, et al. A randomized trial of dialectical behavior therapy versus general psychiatric management for borderline personality disorder. Am J Psychiatr. 2009; 166(12):1365-74.

17. Verheul R, van den Bosch LM, Koeter MW, De Ridder MA, Stijnen T, Van Den Brink W. Dialectical behaviour therapy for women with borderline personality disorder: 12-month, randomized clinical trial in the Netherlands. Br J Psychiatry. 2003;182:135-40.

18. Linehan MM, Heard HL, Armstrong HE. Naturalistic follow-up of a behavioral treatment for chronically suicidal parasuicidal borderline patients. Arch Gen Psychiatry. 1993;50(12):971-4

19. McMain SF, Guimond T, Streiner DL, Cardish RJ, Links PS. Dialectical behavior therapy compared with general psychiatric management for borderline personality disorder: clinical outcomes and functioning over a 2-year follow-up. Am J Psychiatry. 2012;169(6):650-61.

20. van den Bosch LMC, Verheul R, Schippers GM, van den Brink W. Dialectical behavior therapy of borderline patients with and without substance use problems: Implementation and long-term effects. Addict Behav. 2002:27(6):911-23.

21. Barnicot K, Savill M, Bhatti N, Priebe S. A pragmatic randomized controlled trial of dialectical behaviour therapy: effects on hospitalisation and posttreatment follow-up. Psychother Psychosom. 2014;83(3):192-3.

22. Feigenbaum J, Fonagy $\mathrm{P}$, Pilling $\mathrm{S}$, Jones $\mathrm{A}$, Wildgoose $\mathrm{A}$, Bebbington PE. A real-world study of the effectiveness of DBT in the UK national health service. Br J Clin Psychol. 2012;51(2):121-41.

23. Koons CR, Robins CJ, Tweed JL, Lynch TR, Gonzalez AA, Morse JQ, et al. Efficacy of dialectical behavior therapy in women veterans with borderline personality disorder. Behav Ther. 2001;32(2):371-90.

24. Priebe S, Bhatti N, Barnicot K, Bremner S, Gaglia A, Katsakou C, et al. Effectiveness and cost-effectiveness of dialectical behaviour therapy for selfharming patients with personality disorder: a pragmatic randomized controlled trial. Psychother Psychosom. 2012;81(6):356-65.

25. Stoffers JM, Völlm BA, Rücker G, Timmer A, Huband N, Lieb K. Psychological therapies for people with borderline personality disorders (review). Cochrane Database Syst Rev. 2012;8:CD005652.

26. Barnicot K, Gonzalez R, McCabe R, Priebe S. Skills use and common treatment processes in dialectical behaviour therapy for borderline personality disorder. J Behav Ther Exp Psychiatry. 2010:52:147-56.

27. Neacsiu AD, Rizvi SL, Linehan MM. Dialectical behavior therapy skills use as a mediator and outcome of treatment for borderline personality disorder. Behav Res Ther. 2010;48(9):832-9.

28. Zanarini MC, Frankenburg FR, Hennen J, Silk KR. Mental health service utilization by borderline personality disorder patients and axis ॥ comparison subjects followed prospectively for 6 years. J Clin Psychiatry. 2004;65(1):28-36

29. Bender DS, Skodol AE, Pagano ME, Dyck IR, Grilo CM, Shea MT, et al. Prospective assessment of treatment use by patients with personality disorders. Psychiatr Serv. 2006:57(2):254-7.

30. Hörz S, Zanarini MC, Frankenburg FR, Reich DB, Fitzmaurice G. Ten-year use of mental health services by patients with borderline personality disorder and with other axis II disorders. Psychiatr Serv. 2010;61(6):612-6.

31. Bloom JM, Woodward EN, Susmaras T, Pantalone D. Use of dialectical behavior therapy in inpatient treatment of borderline personality disorder: a systematic review. Psychiatr Serv. 2012;63(9):881-8.

32. van den Bosch LMC, Sinnaeve R, Hakkaart-van Roijen $L$, van Furth EF. Efficacy and cost-effectiveness of an experimental short-term inpatient dialectical behavior therapy (DBT) program: study protocol for a randomized controlled trial. Trials. 2014;15:152. https://doi.org/10.1186/ 1745-6215-15-152

33. Thornicroft G, Tansella M. Balancing community-based and hospital-based mental health care. World Psychiatry. 2002:1(2):84-90.

34. Bohus M, Haaf B, Simms T, Limberger MF, Schmahl C, Unckel C, et al. Effectiveness of inpatient dialectical behavior therapy for borderline personality disorder: a controlled trial. Behav Res Ther. 2004;42(5):487-99. 
35. Kröger C, Schweiger U, Sipos V, Arnold R, Kahl KG, Schunert T, et al. Effectiveness of dialectical behaviour therapy for borderline personality disorder in an inpatient setting. Behav Res Ther. 2006;44(8):1211-7.

36. Duijsens IJ, Eurelings-Bontekoe EHM, Diekstra RFW. The VKP, a self-report instrument for DSM-III-R and CD-10 personality disorders: construction and psychometric properties. Personal Individ Differ. 1996;20(2):171-82.

37. Van Vliet I, de Beurs E. Het Mini Internationaal Neuropsychiatrisch Interview (MINI). Een kort gestructureerd diagnostisch psychiatrisch interview voor DSM-IV- en ICD-10-stoornissen. Tijdschr Psychiatr. 2007;49(6):393-7.

38. Weertman A, Arntz A, Dreessen L, van Velzen C, Vertommen S. Shortinterval test-retest interrater reliability of the Dutch version of the structured clinical interview for DSM-IV personality disorders (SCID-II). J Personal Disord. 2003;17(6):562-7.

39. Giesen-Bloo JH, Wachters LM, Schouten E, Arntz A. The borderline personality disorder severity index-IV: psychometric evaluation and dimensional structure. Personal Individ Differ. 2010;49(2):136-41.

40. Brooks R. EuroQol: the current state of play. Health Policy. 1996;37(1):53-72.

41. Lamers LM, McDonnell J, Stalmeier PFM, Krabbe PF, Busschbach JJ. The Dutch tariff: results and arguments for an effective design for national EQ5D valuation studies. Health Econ. 2006;15(10):1121-32.

42. Bouwmans C, De Jong K, Timman R, Zijlstra-Vlasveld M, Van der FeltzCornelis C, Tan Swan S, Hakkaart-van Roijen L. Feasibility, reliability and validity of a questionnaire on healthcare consumption and productivity loss in patients with a psychiatric disorder (TiC-P). BMC Health Serv Res. 2013;13:1

43. Hakkaart-van Roijen L, van der Linden N, Bouwmans C, Kanters T, Tan SS. Kostenhandleiding: Methodologie van kostenonderzoek en referentieprijzen voor economische evaluaties in de gezondheidszorg. Rotterdam: Institute for Medical Technology Assessment; 2015. Available from: https://www. zorginstituutnederland.nl/publicaties/publicatie/2016/02/29/richtlijn-voorhet-uitvoeren-van-economische-evaluaties-in-de-gezondheidszorg

44. Molenberghs G, Verbeke G. Models for discrete longitudinal data. New York: Springer; 2006.

45. Molenberghs G, Kenward M. Missing data in clinical studies, vol. 61. Chichester: Wiley; 2007.

46. Swaap J, Knies S, van der Meijden C, Staal P, van der Heiden L. Kosteneffectiviteit in De Praktijk. Diemen: Zorginstituut Nederland; 2015. Available from https://www.zorginstituutnederland.nl/publicaties/rapport/ 2015/06/26/kosteneffectiviteit-in-de-praktijk

47. Kleindienst N, Limberger MF, Schmal C, Steil R, Ebner-Priemer UW, Bohus M. Do improvements after inpatient dialectical behavior therapy persist in the long term? A naturalistic follow-up in patients with borderline personality disorder. J Nerv Ment Dis. 2008;196(11):847-51.

48. Torgerson DJ, Roland M. What is Zelen's design? Br Med J. 1998;316(7131):606.

\section{Ready to submit your research? Choose BMC and benefit from:}

- fast, convenient online submission

- thorough peer review by experienced researchers in your field

- rapid publication on acceptance

- support for research data, including large and complex data types

- gold Open Access which fosters wider collaboration and increased citations - maximum visibility for your research: over $100 \mathrm{M}$ website views per year

At BMC, research is always in progress.

Learn more biomedcentral.com/submissions 